\title{
LeGes
}

Sarah Vittoz / Karl-Marc Wyss

\section{Forum für Rechtsetzung vom 24. Juni 2021}

\section{Lobbyismus in der Bundesverwaltung, geteilte Umsetzung des Bundesrechts und Regulierungs- folgenabschätzung}

Tagungsbericht zum 37. Forum für Rechtsetzung vom 24. Juni 2021. Die folgenden drei Themen standen auf dem Programm: Lobbyismus in der Bundesverwaltung, geteilte Umsetzung des Bundesrechts sowie die Regulierungsfolgenabschätzung.

Beitragsart: Tagungsberichte

Zitiervorschlag: Sarah Vittoz / Karl-Marc Wyss, 37. Forum für Rechtsetzung vom 24. Juni 2021, in: LeGes 32 (2021) 3 
[1] Im ersten Referat des Nachmittags setzte sich MarTIN HILTI (Geschäftsführer Transparency International Schweiz) mit der Qualität und der Legitimität des Lobbyings in der Bundesverwaltung auseinander. Lobbying ist die gezielte Einflussnahme auf die Willensbildung von Entscheidungsträgerinnen und Entscheidungsträgern in Politik und Verwaltung. Nach dieser Definition verwies Hitri einleitend auf das bestehende Spannungsfeld beim Lobbyismus zwischen den legitimen Interessenseinbezug und der ungebührlichen Einflussnahme. Es sei unbestritten, dass bei Regierung und Verwaltung lobbyiert werde und dies auch wirke. So habe man z.B. in der Pandemie sehen können, welche Branchenverbände und Interessengruppe bei der Regierung mehr Gehör fanden als andere, was sich dann teilweise in den Massnahmen der Covid-19-Gesetzgebung widerspiegelte. Der Umgang mit Lobbyismus in Regierung und Verwaltung ist jedoch kaum reguliert. So kennt die Schweiz keine umfassende Lobbying-Gesetzgebung, sondern bloss einige wenige punktuelle Regeln. Klare und verbindliche Rahmenbedingungen fehlen.

[2] Um einen demokratischen Meinungsbildungsprozess zu gewährleisten, sollte Lobbying gewisse Legitimitätskriterien berücksichtigen. Transparency International Schweiz hat dazu einen mehrstufigen Kriterienkatalog entwickelt für ein offenes und demokratiepolitisch erwünschtes Lobbying, das auch den OECD-Grundsätzen für Transparenz und Integrität in der Lobbyarbeit entspricht. Drei Punkte stehen dabei im Vordergrund: (i.) Transparenz: legislativer Fussabdruck, Informationszugang und Öffentlichkeitsprinzip, Offenlegung von Interessenbindungen und Interessenkonflikten (Ausstandskonstellationen), Registrierung und Offenlegungspflichten von Lobbyistinnen und Lobbyisten; (ii.) Integrität: Verhaltenskodex für Amtspersonen und Verwaltungsangestellte, robuste Selbstregulierung der Branche; (iii.) Zugang: Chancengleicher Zugang zu Entscheidungsträgerinnen und -trägern sowie Entscheidungsorganen, ausgewogene Zusammensetzung von Arbeitsgruppen.

[3] Angesichts der obigen Ausführungen sah Hitrı primär in vier Punkten Handlungsbedarf bei der Bundesverwaltung: Erstens sei der legislative Fussabdruck offenzulegen. Dafür sollten die wesentlichen Einflussnahmen durch Lobbyierende erfasst werden. Ziel wäre eine konsequent geführte, öffentlich zugängliche Dokumentation, wo, wann und mit welchen Eingaben Lobbyierende auf den politischen Meinungsbildungsprozess einwirken. Zu informieren wäre z.B. über die Zusammensetzung von Arbeitsgruppen und relevante schriftliche Eingaben. Erst dieser legislative Fussabdruck erlaubt es der Bevölkerung, die gesetzgeberischen Arbeiten von Regierung, Parlament und Verwaltung verstehen und beurteilen zu können. Zweitens sollte die Bundesverwaltung auf einen chancengleichen Zugang zur Verwaltung achten. So wären z.B. Expertenund Beratungsgremien (Arbeitsgruppen) ausgewogen zusammenzustellen. Drittens bräuchte es eingehendere Regeln bei Seitenwechsel von Bundesräten und oberen Bundeskader in die Privatwirtschaft («Cooling-off»-Periode - Karenzfristen), wenn öffentliche Interessen aufgrund des Wechsels zu Schaden kommen könnten. Viertens seien die Regelungen zum Umgang mit Geschenken zu überarbeiten. Für weiter Informationen und Ausführungen verwies HiLti auf den Bericht «LOBBYING IN DER SCHWEIZ, Verdeckter Einfluss - Heikle Verflechtungen - Privilegierter Zugang» von Transparency International Schweiz vom Februar 2019.

[4] Das zweite Referat hielt Reto Wiesli (Präsident der Schweizerischen Public Affairs Gesellschaft / Partner bei der Politik- und Kommunikationsberatungsagentur polsan AG) zum Zugang zur Verwaltung und Offenheit der Verwaltung für die spezifischen Bedürfnisse von Public Affairs Beauftragten. Er stellt kurz die Schweizerische Public Affairs Gesellschaft (SPAG) und ihre Ziele vor. Nach dem Scheitern gesetzgeberischer Ansätze stand für die SPAG die Selbstdeklaration (Selbstregulierung) und die Weiterbildung ihrer Mitglieder im Vordergrund. So existie- 
ren beispielsweise eine Arbeitsgruppe zu Standesregeln, eine neue Standeskommission unter dem Präsidium von Hanspeter Thür sowie eine obligatorische Weiterbildung für SPAG-Neumitglieder. Anschliessend stellte WiEsLi vor, wie sich Interessen in der Schweiz organisieren und einbringen. Dazu verwies er u.a. auf die verschiedenen Modelle der pluralistischen Interessenvermittlung im Staat: Zugang zur Macht nach gruppenpluralistischem Demokratiemodell und nach ressourcentheoretischen Überlegungen (schwache versus starke Interessen). Den Schweizer Entscheidprozess und die verschiedenen Einflussnahmemöglichkeiten erläuterte WiesLı anhand des Policy Cycle und des Vierphasen-Modells von Linder. Interessant ist der Vergleich mit unseren Nachbarstaaten. Gemäss OECD-Recommendation of the Council on Principles for Transparency and Integrity in Lobbying, Nr. 7 sollten Länder eine Kultur der Integrität in öffentlichen Organisationen und bei der Entscheidungsfindung fördern, indem sie klare Regeln und Leitlinien für das Verhalten öffentlicher Bediensteter festlegen. Die Schweiz schneidet bezüglich Transparenz der Lobbyaktivitäten relativ schlecht ab. WiEsLi stellte dazu den formellen Zugang - z.B. zu Dokumenten über Art. 8 Öffentlichkeitsgesetz (BGÖ; SR 152.3) dem informellen Kontakt gegenüber und verwies auf die Idee offener Agenden, wie sie z.B. in Spanien oder in Grossbritannien existieren. Dort sind Treffen von Regierungs- und Verwaltungsvertreterinnen und -vertretern offenzulegen. Etwas Sorgen bereitet der SPAG die Tendenz der Beschleunigung des Gesetzgebungsverfahrens und die ständigen Neuanfänge bei Projekten. Gerade amtsjunge Parlamentarierinnen und Parlamentarier kennen oft nur noch den beschleunigten Betrieb angesichts der Corona-Krise, was die Tendenz zu unausgereiften Projekten verstärken dürfte. Teilweise stellt sich zudem die Frage, ob die Medien ihre Aufgabe als vierte Gewalt im Staat noch hinreichend wahrnehmen können.

[5] In der anschliessenden rund halbstündigen Podiumsdiskussion unter der Leitung von Monique Cossali Sauvain (Leiterin Fachbereich Rechtsetzungsprojekte I, St. Leiterin Direktionsbereich ÖFFR, Bundesamt für Justiz) diskutierten neben den genannten Referenten auch MicheL Besson (Leiter Fachbereich Rechtsetzungsprojekte II, Bundesamt für Justiz) und Oliver Zibung (Mitarbeiter des Rechtsdiensts, Staatssekretariat für internationale Finanzfragen): So sprach Cossali Sauvain u.a. den Begriff «Transparenz» beim Lobbying an und erkundigte sich nach den konkreten Auswirkungen, welche das Prinzip der Transparenz auf die Aktivitäten der Bundesverwaltung haben sollte. Zur Debatte stellte sie zudem die Frage, wie sich eine Waffengleichheit zwischen den verschiedenen Interessengruppen gewährleisten liesse. Hitrı betonte die Wichtigkeit der Transparenz - es handelt sich um ein Kardinalsprinzip bei der Arbeit der Verwaltung mit Lobbyistinnen und Lobbyisten. So seien z.B. die Arbeitsgruppen nicht nur möglichst ausgeglichen zu besetzen, sondern die Besetzung auch transparent in Berichten der Bundesverwaltung auszuweisen. Aber auch die Verbindungen von Parlamentarierinnen und Parlamentariern seien besser offenzulegen. Wiesti verwies bezüglich der Forderung nach Offenlegung der parlamentarischen Aktivitäten auf die praktikablen Beispiele der offenen Agenden, wie sie in andern europäischen Staaten existieren. Von Seite der Verwaltung wies Zibung darauf hin, dass sie im Staatssekretariats für internationale Finanzfragen grundsätzlich gute Erfahrungen gemacht hätten mit dem Einbezug von Branchenvertreterinnen und -vertretern. Gerade bei Regulierungsfragen sei der Austausch mit verschiedenen Gruppierungen hilfreich gewesen und habe den Fokus auf die Fragen der Praxis geschärft. Auch gemäss Besson verschliesst sich die Verwaltung keineswegs dem Austausch mit der Praxis und wehrt sich nicht gegen mehr Transparenz. Er habe z.B. bei der Erarbeitung des Geldspielgesetzes (BGS; SR 935.51) vielmehr gute Erfahrungen mit Branchenvertreterinnen und -vertretern und Interessengruppen gemacht. Es sei jedoch wichtig, ein Bewusstsein dafür zu schaffen. Namentlich in der Konsultationsphase sowie bei der Zusam- 
mensetzung der Arbeits- und Sachverständigengruppen könne die Verwaltung für Transparenz und bessere Waffengleichheit sorgen.

[6] Nach der Pause stellte Rebecca Joly (Stellvertretende Generalsekretärin des Schweizerischen Mieterinnen- und Mieterverbands) im dritten Referat ihre Dissertation vor, die 2020 bei Helbing Lichtenhahn erschienen war (ReBecCa Joly, La mise en œuvre partagée du droit en Suisse, en Allemagne et dans l'Union européenne, Basel 2020). Die Dissertation behandelt die geteilte Umsetzung des Rechts in der Schweiz, Deutschland und der Europäischen Union. Die geteilte Umsetzung unterscheidet sich von der «Ausführung» oder «Durchsetzung» des Gesetzes oder vom «Vollzugsföderalismus». Im Schweizer Recht ist die geteilte Umsetzung am deutlichsten, wenn die Bundesversammlung ein Bundesgesetz verabschiedet und die Kantone dieses umsetzen müssen. Das System der geteilten Umsetzung hat mehrere Vorteile: Es erlaubt erstens, mehrere Behördenebenen - Bund, Kantone und sogar Gemeinden - in die Umsetzung miteinzubeziehen. Zweitens erleichtert es die Übertragung von Kompetenzen zwischen diesen Ebenen. Drittens führt es zu einer effizienten Nutzung der verfügbaren Ressourcen, da sich Standards aus allen Regierungsebenen koordiniert auf einer Ebene umsetzen lassen. Dies war z.B. der Fall beim Schutz der Biodiversität - einem Anwendungsbereich, den die Dissertation vertieft behandelt. Allerdings müssen die ausführenden Behörden (z.B. der Kantone) bereit sein, die Bundesnormen umzusetzen. So besteht das Risiko einer mangelhaften Umsetzung, namentlich bei politischen Widerständen auf den unteren Ebenen (Kantone und Gemeinden). Zudem kann die geteilte Umsetzung die Möglichkeit, Normen dem lokalen Kontext anzupassen, mehr oder weniger einschränken.

[7] Die Arbeit der Kantone bei der Umsetzung des Bundesrechts ist essentiell. Im Fokus stehen daher verschiedene Massnahmen, um Probleme bei der geteilten Umsetzung zu vermeiden: So ist auf Bundesebene bereits im Vorfeld der Umsetzung des betroffenen Bundesrechts eine Analyse durchzuführen - also bereits zum Zeitpunkt seiner Verabschiedung. Diese Analyse sollte dabei den gewünschten Grad der Zentralisierung in dem betreffenden Bereich definieren. Zudem sollte der Bund die Kantone so früh wie möglich in den Prozess einbeziehen, da der politische Konsens eine wichtige Grundlage für eine optimale Umsetzung bildet. In späteren Phasen der Umsetzung sollte die Bundesebene weiterhin involviert und aktiv bleiben und namentlich Verwaltungsverordnungen erlassen oder finanzielle Unterstützung leisten. Gerade knappe oder fehlende - insbesondere finanzielle - Ressourcen in den Kantonen bieten bei der geteilten Umsetzung häufig Probleme in der Praxis. So verfügen die - oder einzelne - Kantone nicht stets über hinreichend Mittel und/oder Fachpersonal, um die Umsetzung sicherzustellen, z.B. betreffend Datenbereitstellung und -verarbeitung. Der Bund sollte daher die Umsetzung begleiten, überwachen und Sanktionen vorsehen, falls die Kantone das Bundesrecht nicht oder nicht hinreichend umsetzen. Dies ist namentlich in Bereichen notwendig, in denen die Umsetzung kostspielige Massnahmen erfordert und wenig öffentliche Unterstützung und Druck existiert, wie z.B. beim Schutz der Biodiversität. Gemäss JoLy liesse sich die geteilte Umsetzung in der Schweiz wie folgt verbessern (Denkanstösse): Erstens sollte der Bund die vorgelagerte Analyse zu den Umsetzungsmodalitäten in den Kantonen verstärken. So könnte er bundesrechtliche Normen und Vorgaben offener ausgestalten, um den Kantonen mehr Spielraum bei der konkreten Umsetzung zu eröffnen. Zweitens sollte er den Kantonen ausreichend Ressourcen, einschliesslich wissenschaftlicher und finanzieller Mittel, für die Umsetzung des Bundesrechts zuweisen. Drittens sollte er ein Sanktionssystem vorsehen, damit er auf Verzögerungen oder eine mangelhafte Umsetzung reagieren kann. Denkbar wäre z.B. der Entzug von Subventionen. Ein derartiges System kennt z.B. die Europäische 
Union. Dieses könnte auch für die Schweiz eine Option sein, um die Umsetzung von Bundesnormen durch die Kantone zu optimieren.

[8] Die anschliessende Diskussion mit Plenumsfragen drehte sich primär um Umsetzung des Bundesrechts durch die Kantone während der Covid-19-Pandemie. So wiesen verschiedene Teilnehmende auf ihre Erfahrungen mit der Umsetzung des Notrechts hin und verdeutlichten dabei bestehende Grenzen und Mängel der geteilten Umsetzung. Die Diskussion zeigte, dass Bund und Kantone ihre Zusammenarbeit vertieft analysieren sollten, um die kantonale Umsetzung von Bundesrecht inklusive Vollzug in Krisenzeiten künftig zu verbessern.

[9] Im abschliessenden Referat ging Frau Annetta Holl (Wissenschaftliche Mitarbeiterin im Fachbereich «Regulierungsanalyse und -politik», Staatssekretariat für Wirtschaft SECO) auf die Regulierungsfolgenabschätzung ein. Einleitend stellte sie kurz vor, was unter Regulierungsfolgenabschätzung (RFA) zu verstehen ist - nämlich ein Instrument im Rechtsetzungsprozess, das analysiert und darstellt, wie sich eine Vorlage des Bundes volkswirtschaftlich auswirkt und wie Alternativen aussehen könnten. Die RFA zielt darauf ab, Transparenz zu schaffen, faktenbasierte Entscheidung zu ermöglichen und Vorlagen zu optimieren. Die Richtlinien des Bundesrates für die Regulierungsfolgenabschätzung bei Rechtsetzungsvorhaben des Bundes (RFA-Richtlinien) vom 6. Dezember 2019 geben dazu fünf Prüfpunkte vor: (i.) Notwendigkeit und Möglichkeit staatlichen Handelns; (ii.) Alternative Handlungsoptionen; (iii.) Auswirkungen auf die einzelnen gesellschaftlichen Gruppen; (iv.) Auswirkungen auf die Gesamtwirtschaft; (v.) Zweckmässigkeit im Vollzug. Die Ämter sollen Alternativen möglichst früh prüfen, weshalb dieser Prüfpunkt neu bereits an zweiter Stelle folgt. Weitere Informationen zu RFA und Prüfpunkten finden sich im RFA-Handbuch und in der RFA-Checkliste. Die Ergebnisse einer RFA fliessen ein in die Anträge an den Bundesrat sowie in die erläuternden Berichte und Botschaften.

[10] Die neuen RFA-Richtlinien sind seit dem 1. Februar 2020 in Kraft und bezwecken, eine einfache rechtzeitige und einheitliche Anwendung der RFA für alle Rechtsetzungsvorhaben des Bundes, wobei die Verantwortung - mit Ausnahme der vertieften RFA - beim federführenden Amt liegt. So muss die Bundesverwaltung für sämtliche Rechtsetzungsvorhaben einen Quick-Check durchführen, der bei der ersten Ämterkonsultation zum Aussprachepapier oder dem Antrag beizulegen ist. Es handelt sich beim Quick-Check um eine Kurzabschätzung der RFA-Prüfpunkte anhand eines Excelformulars des WBF. Als Relevanzanalyse hilft er die Art und den Umfang der RFA zu ermitteln. Zu prüfen sind die fünf Prüfpunkte dann entsprechend im Bericht des federführenden Amtes (interne RFA), dem Bericht eines externen Auftragnehmers (externe RFA) oder im Bericht, den ein externer Auftragnehmer zusammen mit der RFA-Fachstelle des SECO erstelle (vertiefte RFA). Eine vertiefte RFA ist bei Vorhaben des Bundes mit mittleren oder starken Auswirkungen auf die Volkswirtschaft oder auf die Unternehmen durchzuführen. Normalerweise schlägt das federführende Departement oder Amt in Absprache mit der RFA-Fachstelle des SECO vor, welche Vorhaben einer vertieften RFA bedürfen. Neu muss sich jedoch auch der Bundesrat in seinen Beschlüssen zur Notwendigkeit einer vertieften RFA äussern. Die RFA ist dazu möglichst früh in die Erarbeitung der Vorlagen einzubeziehen, so z.B. im Gesetzgebungsverfahren (Grafik nach Annetta Holl, Folie 12 des Referats): 


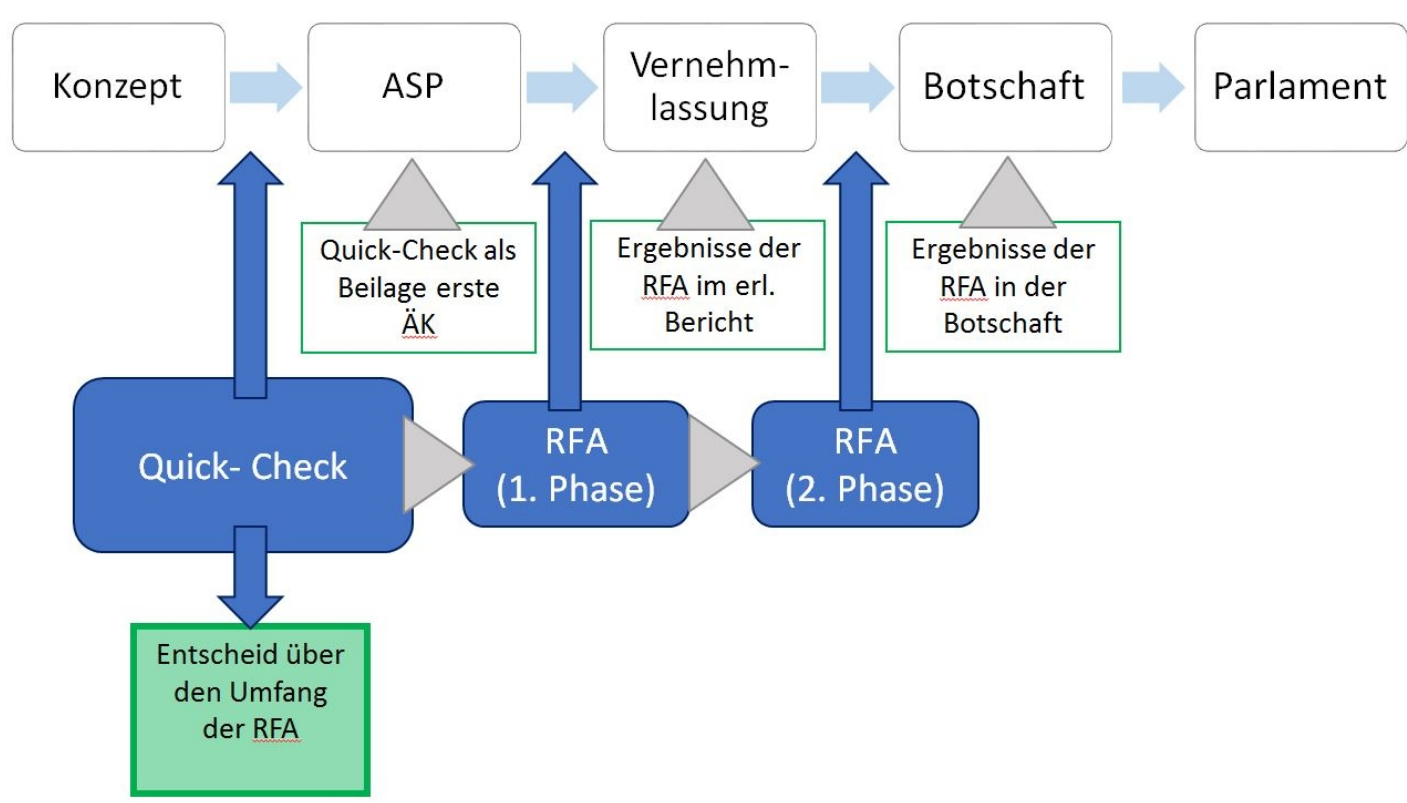

[11] Nach einer Übergangsphase wenden die Ämter den Quick-Check nun an, wobei sie diesen als grundsätzlich sinnvoll erachten. Die Praxiserfahrung bei Ämtern und SECO entwickelt sich schrittweise. Entsprechend sieht sich die RFA-Fachstelle des SECO noch mit vielen formellen und materiellen Fragen zu bestimmten Vorlagen und Inhalten konfrontiert. So stellten sich zahlreiche Fragen zum Anwendungsbereich, z.B. betreffend völkerrechtliche Verträge, die einen Genehmigungsbeschluss im Parlament benötigen, zu Gegenentwurf und Gegenvorschlag bei Volksinitiativen sowie zur dringlichen Gesetzgebung (Covid-Verordnungen). Von der Seite RFA-Fachstelle haben wir zu Verständlichkeit und Qualität der Aussagen der Ämter in der RFA festgestellt, dass diese zu Handlungsbedarf und Zielen oft nur formelle statt inhaltliche Aussagen angeben. So finden sich oft Verweise "gemäss Auftrag vom...» oder so ähnlich. Dagegen umschreiben die Ämter die Auswirkungen jeweils meist gut. Zurzeit wertet das SECO die Anwendung des Quick-Checks erstmal aus und erstellt eine Zwischenbilanz. Zudem wird es die methodischen Hilfsmittel überarbeiten (Handbuch, Checkliste). Nach vier Jahren und damit im Jahre 2024 findet eine Evaluation der gesamten RFA-Richtlinien statt.

[12] Sämtliche Tagungsunterlagen finden sich auf der Homepage des Bundesamts für Justiz; abrufbar unter: https://www.bj.admin.ch/bj/de/home/staat/legistik/rechtsetzungsforum/veran staltungsthemen/37.html. Das 38. Forum für Rechtsetzung findet am 16. November 2021 statt und dreht sich thematisch um die parlamentarische Initiative: Arbeit der Kommissionen und Rolle der Verwaltung.

Sarah Vittoz, MLaw, wissenschaftliche Mitarbeiterin, Fachbereich Rechtsetzungsprojekte II, Bundesamt für Justiz.

Dr. iur. KarL-Marc Wyss, Rechtsanwalt, wissenschaftlicher Mitarbeiter, Fachbereich Rechtsetzungsprojekte II, Bundesamt für Justiz. 